Pacific Journal of Mathematics

UNITARY INVARIANTS FOR NESTS 


\title{
UNITARY INVARIANTS FOR NESTS
}

\author{
J. A. ERDos
}

\begin{abstract}
A set of subspaces of a Hilbert space is called a nest if it is totally ordered by inclusion. The aim of this paper is to obtain a complete set of unitary invariants for a class of nests satisfying certain conditions. The conditions imposed are a countability condition, (satisfied by all nests of subspaces of a separable Hilbert space), and a simple completeness condition.
\end{abstract}

As the orthogonal projections onto the members of a nest form a set of commuting projections, the problem has much in common with the invariant theory of self-adjoint operators on Hilbert space and a considerable part of this paper consists of reworking standard multiplicity theory to suit the new situation.

However there is a major difference between the two theories. The spectral projections of a self-adjoint operator can be indexed in a unique way by the real numbers belonging to its spectrum but for nests there is no such indexing that is in any sense canonical. This difficulty is overcome by dispensing with numerical indexing and using instead the set of subspaces of the nest considered as a topological space under its order topology. In this way the order structure of nests is fitted into the invariants.

The problem has been solved for a special case, (nests which have uniform multiplicity one), by R. V. Kadison and I. M. Singer ([4) $\S 3.4)$. Here the above difficulty was overcome by considering a class of subsets of the real line which can be used to index the projections and the order isomorphisms between members of this class which carry sets of zero Lebesgue measure onto these sets of the image. It is felt that this would prove rather cumbersome in the general case.

1. Preliminaries and notation. Throughout this paper, the terms Hilbert space, subspace and projection will be used to mean complex Hilbert space, closed subspace and orthogonal projection respectively. The set of all bounded linear operators on a Hilbert space $H$ will be denoted by $\mathscr{L}(H)$. The orthogonal complement of a subspace $N$ of $H$ will be denoted by $H \ominus N$. The symbol $\oplus$ will always denote orthogonal direct sum. If $N$ is a subspace of $H$ and $E$ is the projection onto $N$, then for any $A \in \mathscr{L}(H)$ the operator of $\mathscr{L}(N)$ formed by restricting the domain of $E A$ to $N$ will be denoted by $A_{N}$ or by $A_{E}$. If $\mathscr{A}$ is any subset of $\mathscr{L}(H)$, the set $\left\{A_{E}: A \in \mathscr{A}\right\}$ 
will be written as $\mathscr{A}_{E}$ or as $\mathscr{A}_{N}$. If $\mathscr{A}$ is a self-adjoint subset of $\mathscr{L}(H)$, (that is, $A \in \mathscr{A}$ implies that $A^{*} \in \mathscr{A}$ ), then the set $\left\{A^{\prime} \in \mathscr{L}(H): A^{\prime} A=A A^{\prime}\right.$ for all $\left.A \in \mathscr{A}\right\}$ is called the commutant of $\mathscr{A}$ and will be denoted by $\mathscr{A}^{\prime}$. We note that a self-adjoint subalgebra $\mathscr{A}$ of $\mathscr{L}(H)$ is a maximal abelian subalgebra of $\mathscr{L}(H)$ if and only if $\mathscr{A}=\mathscr{A}$. A separating vector for $\mathscr{A}$ is a vector of $H$ which is annihilated by no operator of $\mathscr{A}$ other than the zero operator. A generating vector for $\mathscr{A}$ is a vector $x$ of $H$ such that the set $\{A x: A \in \mathscr{A}\}$ is dense in $H$.

The topologies on $\mathscr{L}(H)$ induced by the sets of seminorms $A \rightarrow|\langle A x, x\rangle|$ and $A \rightarrow\|A x\|$ for all $x \in H$, are called the weak and strong operator topologies respectively. A weakly closed self-adjoint subalgebra of $\mathscr{L}(H)$ is called a $W^{*}$-algebra. A well known result due to von Neumann (see e.g. [1] Chapter I, §§3,4) shows that if $\mathscr{A}$ is a self-adjoint subset of $\mathscr{L}(H)$ containing the identity operator then the $W^{*}$-algebra generated by $\mathscr{A}$ is $\mathscr{A}^{\prime \prime}$. If $\mathscr{A}$ is a $W^{*}$ algebra and every set of mutually orthogonal projections of $\mathscr{A}$ is countable, then $\mathscr{A}$ is said to be countably decomposable. Clearly if $H$ is separable, every $W^{*}$-subalgebra of $\mathscr{L}(H)$ is countably decomposable. The following results are stated for reference. Proofs may be found in [1] (for (i), p. 6 and p. 20, and for (ii), p. 89).

THEOREM 1.1. Suppose. is an abelian $W^{*}$ subalgebra of $\mathscr{L}(H)$. Then

(i) $\mathscr{A}$ is countably decomposable if and only if $\mathscr{A}$ has a separating vector.

(ii) If there exists a generating vector for. $\mathscr{A}$ then $\mathscr{A}$ is maximal abelian.

In measure theory we shall follow the definitions and terminology given in [2]. However, as we shall be concerned with compact spaces, we note that in this case the Borel sets are the elements of the $\sigma$ ring generated by all closed (or all open) subsets, and all Borel measures are finite. We use the symbol "œ" for set inclusion, " $\subset$ " being reserved for proper inclusion. The symbol " $\backslash$ " denotes set theoretic difference and the characteristic function of a set $S$ will be denoted by $\chi_{s}$.

A set $\mathscr{N}$ of subspaces of a Hilbert space $H$ is called a complete nest if:

(i) it is totally ordered by inclusion,

(ii) (0) and $H$ are members of $\mathscr{N}$,

(iii) given any subset $\mathscr{N}_{0}$ of $\mathscr{N}$, the subspaces $\bigcap\left\{N: N \in \mathscr{N}_{0}\right\}$, $\operatorname{cl}\left[\bigcup\left\{N: N \in \mathscr{N}_{0}\right\}\right]$ are both members of $\mathscr{N}$.

If $\mathscr{N}$ is a complete nest and $N \in \mathscr{N}, N \neq(0)$, we define $N_{-}$by 


$$
N_{-}=\operatorname{cl}[\bigcup\{M: M \in \mathscr{N}, M \subset N\}] .
$$

Then $N_{-} \in \mathscr{N}$. If $N_{-} \neq N$, we call $N_{-}$the immediate predecessor of $N$ (in $\mathscr{N}$ ). (The above definitions are reproduced from [8].)

Let $\mathscr{E}$ be the set of projections onto the members of a complete nest $\mathscr{N}$. If $F$ is a projection and $F \in \mathscr{E}^{\prime}$, then $\mathscr{E}_{F}$ is a totally ordered set of projections whose ranges clearly form a complete nest of subspaces of the range of $F$. We shall denote this nest by $\mathscr{N}_{F}$. The weak closure of the algebra generated by $\mathscr{E}$ (that is, $\mathscr{E}^{\prime \prime}$ ), will be called the core of $\mathscr{N}(\mathrm{cf}$. [4]). A nest $\mathscr{N}$ is defined to be countably decomposable if its core is countably decomposable.

We obtain a complete set of unitary invariants for countably decomposable complete nests. It is a trivial consequence of results in [8] (Lemma 3.2 and Theorem 3.4) that this will also be a complete set of invariants for the corresponding nest algebras.

2. Complete nests as ordered sets. An ordered set $\{\omega,<\}$ is a set of elements $\omega=\{a, b, \cdots\}$ which are totally ordered by the relation $<$. When the relation is understood we shall speak of $\omega$ as an ordered set. The relation $\leqq$ is defined in the natural way. The subsets of $\omega$ defined by $\{x: a<x<b\},\{x: a \leqq x<b\},\{x: a<x \leqq b\}$ and $\{x: a \leqq x \leqq b\}$ will be denoted by $(a, b),[a, b),(a, b]$ and $[a, b]$ respectively. We establish terminology for ordered sets following Kamke [5] and Kelley [6] (p. 58 problem I). If there exists an element $a_{0}$ in $\omega$ such that $a_{0}<a$ for all $a \in \omega \backslash a_{0}$ then $a_{0}$ is called the first element of $\omega$. Similarly if $a<a_{1}$ for all $a \in \omega \backslash \alpha_{1}$ then $a_{1}$ is called the last element of $\omega$.

Two ordered sets $\{\omega,<\},\{\Omega, \prec\}$ are order isomorphic if there exists a one to one map $\varphi$ from $\omega$ onto $\Omega$ such that $a<b$ if and only if $\varphi(a) \prec \varphi(b)$. Such a map $\varphi$ is called an order isomorphism. A subset $\eta$ of $\omega$ is called order dense if, for arbitrary $a, b \in \omega$ with $a<b$, there exists an element $c$ of $\eta$ such that $a<c<b$. A subset $\alpha$ of $\omega$ is said to be order bounded if there exist elements $b, c$ of $\omega$ such that, for all $a \in \alpha, b \leqq a \leqq c$. If every order bounded subset of an ordered set $\omega$ has a supremum and an infimum then $\omega$ is said to be order complete. If $\omega$ is order complete, for each element $a \in \omega$ (except for the first element of $\omega$ if it exists) we denote by $a_{-}$the element $\sup \{x: x<a\}$. If $a_{-}$is distinct from $a$, it is called the immediate predecessor of $a$. The following theorem follows easily from the theory of ordered sets.

THEOREM 2.1. If $\{\omega,<\}$ is an ordered set such that

(i) $\omega$ has a first and a last element,

(ii) $\omega$ is order complete, 
(iii) $\omega$ has a countable order dense subset. Then $\omega$ is order isomorphic to the real numbers of the closed interval $[0,1]$ ordered by the natural ordering.

Proof. Note that the property of being order complete is equivalent (in the terminology used e.g. in [5]) to the property of having no gaps. The theorem is then a trivial deduction from Theorem 3 p. 77 of [5].

If $\{\omega,<\}$ is an ordered set, the sets $(a, b)$ for all $a, b \in \omega$ form a base for a topology on $\omega$. This topology is called the order topology. If two ordered sets are order isomorphic then their order topologies are homeomorphic.

From the definition of a complete nest $\mathscr{N}$, it is clear that $\{\mathscr{N}, \subset\}$ is an order complete ordered set with a first and a last element, and is homeomorphic under the order topology to $\{\mathscr{E},<\}$ where $\mathscr{E}$ is the set of projections onto the members of $\mathscr{N}$.

THEOREM 2.2. The following statements are equivalent.

(i) The ordered set $\omega$ is order isomorphic to some countably decomposable complete nest $\mathscr{N}$.

(ii) The ordered set $\omega$ is compact and metrisable in the order topology.

(iii) The ordered set $\omega$ is order isomorphic to the points of some closed subset $S$ of $[0,1]$ containing 0 and 1 and ordered by the natural ordering.

Proof. (i) $\Rightarrow$ (ii). If $\omega$ is order isomorphic to the nest $\mathscr{N}$, it follows, from the completeness of $\mathscr{N}$, as in [6] (p. 162 problem C), that $\mathscr{N}$ is compact under the order topology. Since $\mathscr{N}$ is countably decomposable, there exists a vector $x$ that is separating for the set $\mathscr{E}$ of projections onto the members of $\mathscr{N}$. It is easy to verify that the metric defined by

$$
d\left(E_{1}, E_{2}\right)=\left\|\left(E_{1}-E_{2}\right) x\right\|
$$

defines the order topology on $\mathscr{E}$. Hence $\mathscr{N}$ is metrisable.

(ii) $\Rightarrow$ (iii). If $\omega$ is compact and metrisable then it has a countable base $\left\{\gamma_{i}: i=1,2, \cdots\right\}$. Let $a_{1}$ be the last element of $\omega$ and let

$$
\alpha=\left\{a: a \in \omega, a_{-} \neq a\right\} \text {. }
$$

Then for each $a \in \alpha,\left[a, a_{1}\right]=\left(a_{-}, a_{1}\right]$ is an open subset of $\omega$ containing $a$. Hence there exists an integer $n(a)$ such that

$$
a \in \gamma_{n(a)} \subseteq\left[a, a_{1}\right] .
$$


Since $a=\min \gamma_{n(a)}$, distinct members of $\alpha$ give rise to distinct members of the base. Hence $\alpha$ is a countable set.

Let $f$ be a function defined on $\omega$ into $\omega \times[0,1]$ by

$$
\begin{aligned}
& f(a)=(a, 0) \text { for } a \in \omega \backslash \alpha . \\
& f(a)=(a, 1) \text { for } a \in \alpha .
\end{aligned}
$$

Let $\omega^{\prime}$ be the set

$$
\{(a, 0): a \in \omega \backslash \alpha\} \cup\{(a, r): a \in \alpha, r \in(0,1]\}
$$

ordered by the dictionary ordering of $\omega \times[0,1]$. It is easy to see that $\omega^{\prime}$ is order complete and has a first and a last element. Since $\omega$ is compact and metrisable, it is separable and thus contains a countable subset $\eta$ which is dense in the order topology. Let $\eta^{\prime}$ be the union of the image of $\eta$ under $f$ and the subset of $\omega^{\prime}$ defined by

$$
\{(a, r): a \in \alpha, r \text { rational, } r \in(0,1]\} \text {. }
$$

Then it is easily verified that $\eta^{\prime}$ is a countable order dense subset of $\omega^{\prime}$. Hence by Theorem 2.1, $\omega^{\prime}$ is order isomorphic to the closed interval $[0,1]$. Let $g$ be the map effecting this order isomorphism. As $f$ is order preserving, we have that $g f$ is an order isomorphism of $\omega$ onto a subset $S$ of $[0,1]$ and as the extreme elements of $\omega^{\prime}$ are in the range of $f, S$ contains 0 and 1 . The complement of $S$ in $[0,1]$ consists of the set

$$
\bigcup\left\{\left(g\left[f\left(a_{-}\right)\right], g[f(a)]: a \in \alpha\right\}\right.
$$

which is a countable union of open intervals. Hence $S$ is a closed subset of $[0,1]$.

(iii) $\Rightarrow$ (i). Let $S$ be a closed subset of $[0,1]$ and consider the projections of $\mathscr{L}\left(L^{2}[0,1]\right)$ corresponding to multiplication of elements of $L^{2}[0,1]$ by $\chi_{[0, \lambda]}$ for $\lambda \in S$. Then, the ranges of these projections form a complete nest in $L^{2}[0,1]$ and are order isomorphic to the points of $S$. Since $L^{2}[0,1]$ is separable this nest is countably decomposable. The preceding theorem characterises the ordered sets that are order isomorphic to some complete countably decomposable nest. It is, however, an open question whether every nest which is compact and metrisable in the order topology is necessarily countably decomposable.

THEOREM 2.3. If $\omega$ is an ordered set, order isomorphic to a closed subset $S$ of $[0,1]$ then the order isomorphism is a homeomorphism when $\omega$ has the order topology and $S$ has the relative topology induced on it by the usual topology of the real line. 
Proof. This is equivalent to proving that the order topology of $S$ and the usual induced topology coincide. Since $S$ is closed, it is easy to show that for arbitrary real numbers $a, b$ the set $(a, b) \cap S$ can be written as $(p, q) \cap S$ where $p$ and $q$ are points of $S$. The theorem then follows from the fact that the sets $\{(a, b) \cap S: a, b$ real $\}$ and $\{(p, q) \cap S: p, q \in S\}$ form bases for the induced topology and the order topology respectively.

CoRollary 2.4. If $\omega$ is as in Theorem 2.3, any subset of $\omega$ which is open in the order topology can be written as a countable disjoint union of open order intervals.

Proof. Using Theorem 2.3, this is an immediate consequence of the similar result for the open subsets of the real line.

3. The measures associated with a nest. For the whole of this section, $\mathscr{N}$ shall denote a complete nest of subspaces of a Hilbert space $H$ such that $\mathscr{N}$ is metrisable in the order topology. Let $\mathbf{R}$ be the ring of subsets of $\mathscr{N}$ generated by all open order intervals of $\mathscr{N}$. Then it is clear that any member $R$ of $\mathbf{R}$ can be written as a finite disjoint union, $\bigcup_{i=1}^{n} R_{i}$, where each $R_{i}$ is either an open order interval $\left(N_{1}, N_{2}\right)$ or a single point $\left\{N_{1}\right\}$. Let $x$ be any vector of $H$ and define the set function $\mu_{x}$ on $\mathbf{R}$ as follows. For open order intervals and single points let

$$
\begin{aligned}
\mu_{x}\left(\left(N_{1}, N_{2}\right)\right) & =\left\langle\left(E_{2-}-E_{1}\right) x, x\right\rangle \\
\mu_{x}\left(\left\{N_{1}\right\}\right) & =\left\langle\left(E_{1}-E_{1-}\right) x, x\right\rangle
\end{aligned}
$$

where $E_{i}, E_{i-}$ are the projections onto the subspaces $N_{i}, N_{i-},(i=1,2)$, and for a general member $R$ of $\mathbf{R}$

$$
\mu_{x}(R)=\sum_{i=1}^{n} \mu_{x}\left(R_{i}\right)
$$

where $U R_{i}$ is the representation of $R$ as above.

LEMma 3.1. $\mu_{x}$ is a countably additive set function on $\mathbf{R}$.

Proof. This proof is a modification of the similar result for the real line, (see e.g. [2] pp. 32-35). Suppose $\left\{R_{i}: i=1,2, \cdots\right\}$ is a countable disjoint set of members of $\mathbf{R}$ and suppose $\bigcup_{1}^{\infty} R_{i}=R \in \mathbf{R}$. It is required to prove that

$$
\mu_{x}(R)=\sum_{1}^{\infty} \mu_{x}\left(R_{i}\right)
$$


Clearly, it is sufficient to consider the case where $R$ and each $R_{i}$ are either single points or open order intervals. The result is obvious if $R$ is a single point.

Suppose $R=\left(N_{1}, N_{2}\right)$ and $E_{1}, E_{2}$ are the projections onto $N_{1}, N_{2}$. As the supremum of an ordered set of projections is a strong limit point of the set, if $N_{2^{-}}=N_{2}$ then for any $\varepsilon>0$, there exists $N_{2}^{\prime} \subset N_{2}$ such that $N_{2}^{\prime} \in \mathscr{N}$ and

$$
\mu_{x}\left(\left(N_{2}^{\prime}, N_{2}\right)\right)<\varepsilon / 3 .
$$

If $N_{2^{-}} \neq N_{2}$, let $N_{2}^{\prime}=N_{2-}$. Similarly there exists $N_{1}^{\prime} \supset N_{1}$ such that

$$
\mu_{x}\left(\left(N_{1}, N_{1}^{\prime}\right)\right)<\varepsilon / 3 .
$$

Thus there is a closed interval $\left[N_{1}^{\prime}, N_{2}^{\prime}\right]$ included in $\left(N_{1}, N_{2}\right)$ such that

$$
\mu_{x}\left(\left(N_{1}, N_{2}\right)\right)-\mu_{x}\left(\left[N_{1}^{\prime}, N_{2}^{\prime}\right]\right)<2 \varepsilon / 3 \text {. }
$$

Similar considerations show that if $R_{i}$ is a single point, it is contained in an open order interval $R_{i}^{\prime}$ such that

$$
\mu_{x}\left(R_{\imath}^{\prime}\right)-\mu_{x}\left(R_{i}\right)<\varepsilon / 3.2^{i} .
$$

If $R_{i}$ is an open interval, let $R_{i}^{\prime}=R_{i}$. Then the set $\left\{R_{i}: i=1,2, \cdots\right\}$ is an open cover of $\left[N_{1}^{\prime}, N_{2}^{\prime}\right]$ and by picking a finite subcover, it follows easily that

$$
\mu_{x}\left(\left(N_{1}, N_{2}\right)\right) \leqq \sum_{1}^{\infty} \mu_{x}\left(R_{\imath}\right)
$$

As the opposite inequality is easy to prove, the lemma follows.

Standard measure theory (see e.g. [2] p. 41 et seq.) shows that $\mu_{x}$ may be extended to a measure on some $\sigma$-ring $S_{x}$. It should cause no confusion if this measure is denoted by $\mu_{x}$. As for each $x \in H$, $S_{x}$ contains all open intervals, and hence by Corollary 2.4, all open sets, it follows from the compactness of $\mathscr{N}$ that each $S_{x}$ contains the Borel sets of $\mathscr{N}$. Hence, for all $x \in H$, as $\mu_{x}$ is clearly finite, $\mu_{x}$ is a Borel measure on $\mathscr{N}$. We shall denote the set of Borel sets of $\mathscr{N}$ by $\mathscr{B}$.

TheOREm 3.2. If $f(N)$ and $g(N)$ are bounded, Borel measurable functions defined on $\mathscr{N}$, there exists a bounded linear operator $T_{f}$ belonging to the core $\mathscr{C}$ of $\mathscr{N}$, such that

$$
\left\langle T_{f} x, x\right\rangle=\int_{\mathscr{r}} f d \mu_{x} .
$$

Also,

(i) $k T_{f}=T_{k f}(k$ a complex number $)$ 
(ii) $T_{f}+T_{g}=T_{f+g}$

(iii) $T_{f} \cdot T_{g}=T_{f \cdot g}$

(iv) $\left\langle T_{f} x, T_{g} x\right\rangle=\int_{\mathscr{r}} f \bar{g} d \mu_{x}$

(v) $T_{f} x=T_{g} x$ if and only if $f=g \mu_{x}$-almost everywhere.

Proof. Define the complex valued measure $\nu_{x, y}$ by

$$
4 \nu_{x, y}=\mu_{x+y}-\mu_{x-y}+i \mu_{x+i y}-i \mu_{x-i y} .
$$

Then, from the definition of $\mu_{x}$, it is clear that for any open order interval $\left(N_{1}, N_{2}\right), \nu_{x, y}\left(\left(N_{1}, N_{2}\right)\right)$ is a bilinear form on $H$. But the relations expressing this bilinearity are equations between linear combinations of measures and therefore they hold on a $\sigma$-ring. As this $\sigma$-ring contains the open intervals, it follows, using Corollary 2.4, that for all Borel sets $\delta$ of $\mathscr{N}, \nu_{x, y}(\delta)$ is a bilinear form on $H$. It is now easy to show that in the equation

$$
\left\langle T_{f} x, y\right\rangle=\int_{\mathscr{r}} f d \nu_{x, y}
$$

the right hand side is a bounded bilinear form on $H$ and hence defines the operator $T_{f}$.

We show that $T_{f} \in \mathscr{C}\left(=\mathscr{C}^{\prime \prime}\right)$. If $A \in \mathscr{C}^{\prime}$, the relation

$$
\nu_{A x, y}(\delta)=\nu_{x, A^{*} y}(\delta)
$$

is easily verified if $\delta$ is an open interval and hence holds for all $\delta \in \mathscr{B}$. Therefore, for all $x, y \in H$,

$$
\begin{aligned}
\left\langle T_{f} A x, y\right\rangle & =\int_{\mathscr{r}} f d \nu_{\Delta x, y} \\
& =\left\langle T_{f} x, A^{*} y\right\rangle \\
& =\left\langle A T_{f} x, y\right\rangle
\end{aligned}
$$

and so $T_{f} \in \mathscr{C}$.

The properties of $T_{f}$ are easily proved using approximations to $f$ and $g$ by simple functions. The details are omitted.

If $\delta$ is a Borel set of $\mathscr{N}$, we denote the operator $T_{\chi_{\delta}}$ by $E(\hat{\delta})$. From Theorem 3.2(iii), $E(\delta)$ is a projection and, if $\delta, \delta^{\prime} \in \mathscr{B}$, $E\left(\delta \cap \delta^{\prime}\right)=E(\delta) \cdot E\left(\delta^{\prime}\right)$. As $\langle E(\cdot) x, x\rangle=\mu_{x}(\cdot)$, it is clear that $E(\cdot)$ is countably additive on $\mathscr{B}$ in the strong operator topology. We call $E(\cdot)$ the projection valued measure for the nest $\mathscr{N}$. We shall denote the range of $E(\delta)$ by $N(\delta)$.

For each $x \in H$ we define the subspace $M(x)$ of $H$ by

$$
M(x)=\operatorname{cl}[\operatorname{span}\{E(\delta) x: \delta \in \mathscr{B}\}]
$$


where $\mathscr{B}$ is the set of Borel sets of $\mathscr{N}$.

LeMma 3.3. (i) $M(x)=M(k x)$ ( $k$ a complex number).

(ii) $M(E(\delta) x)=N(\delta) \cap M(x)$.

(iii) If $E\left(\delta_{1} \cap \delta_{2}\right)=0$ and $x_{i} \in N\left(\delta_{i}\right)(i=1,2$, then

$$
M\left(x_{1}+x_{2}\right)=M\left(x_{1}\right) \oplus M\left(x_{2}\right) .
$$

(iv) The projection onto $M(x)$ belongs to the commutant $\mathscr{C}^{\prime}$ of the core $\mathscr{C}$ of $\mathscr{N}$.

Proof. (i), (ii) and (iii) are simple consequences of the definition of $M(x)$. To prove (iv), we note that if $\mathscr{E}$ is the set of projections onto the members of $\mathscr{N}, M(x)$ is invariant under each member of $\mathscr{E}$. Hence, if $P$ is the projection onto $M(x)$, for all $E \in \mathscr{E}$,

$$
\begin{aligned}
E P & =P E P \\
& =(P E P)^{*} \\
& =P E
\end{aligned}
$$

and hence $P \in \mathscr{E}^{\prime}=\mathscr{C}^{\prime}$.

If $\mu$ is a measure on $\mathscr{N}$, we denote by $L^{2}(\mathscr{N}, \mu)$ the Hilbert space of functions $f$ defined on $\mathscr{N}$ such that $\int_{\mathscr{r}}|f|^{2} d \mu$ exists and is finite.

THEOREM 3.4. There exists a unitary transformation $U$ taking $M(x)$ onto $L^{2}\left(\mathscr{N}, \mu_{x}\right)$, such that for any Borel subset $\delta$ of $\mathscr{N}$, $U E(\delta) U^{*}$ is the operator on $L^{2}\left(\mathscr{N}, \mu_{x}\right)$ which multiplies the functions of $L^{2}\left(\mathscr{N}, \mu_{x}\right)$ by $\chi_{\delta}$.

Proof. Using the notation of Theorem 3.2,

$$
M(x)=\operatorname{cl}\left\{T_{f} x: f \text { a simple function }\right\} .
$$

The transformation $T_{f} x \rightarrow f$, by Theorem 3.2, (i), (ii), (iv) and (v), is a linear, isometric and one to one transformation from a dense subset of $M(x)$ onto a dense subset of $L^{2}\left(\mathscr{N}, \mu_{x}\right)$. Hence it may be extended by continuity to a unitary transformation.

If $f$ is a bounded, Borel measurable function,

$$
\begin{aligned}
U E(\delta) U^{*} f & =U T_{x_{\delta}} \cdot T_{f} x \\
& =\chi_{\delta} \cdot f .
\end{aligned}
$$

But $E(\delta)$ is a bounded operator and the bounded, Borel measurable functions form a dense subset of $L^{2}\left(\mathscr{N}, \mu_{x}\right)$. Therefore the above relation holds for all $f \in L^{2}\left(\mathscr{N}, \mu_{x}\right)$.

Corollary 3.5. $M(x)$ is separable. 
Proof. As $\mathscr{N}$ is compact and metrisable, it is separable and therefore, if $\left\{N_{i}: i=1,2, \cdots\right\}$ is a countable dense subset of $\mathscr{N}$, it is easy to see that the smallest $\sigma$-ring containing all intervals of the form $\left(N_{i}, N_{j}\right)$ consists of the Borel sets of $\mathscr{N}$. The fact that $L^{2}\left(\mathscr{N}, \mu_{x}\right)$ is separable now follows from [2], (Theorem B, p. 168 and Problem (1) p. 177).

As in [2], we write $\mu_{1} \sim \mu_{2}$ if $\mu_{1}$ and $\mu_{2}$ are equivalent measures, and $\mu_{1} \prec \mu_{2}$ if $\mu_{1}$ is absolutely continuous with respect to $\mu_{2}$.

THeOREM 3.6. If $\mu$ is a Borel measure on $\mathscr{N}$ and, for some $x \in H, \quad \mu \prec \mu_{x}$, then there exists a vector $y \in H$ such $\mu=\mu_{y}$. If $\mu \sim \mu_{x}$ then $y$ may be chosen so that $M(x)=M(y)$.

Proof. As $\mu \prec \mu_{x}$, by the Radon-Nikodym Theorem (see e.g. [2] p. 128), there is a real valued, positive function $h$ defined on $\mathscr{N}$ such that

$$
\mu(\delta)=\int_{\delta} h(N) d \mu_{x}
$$

for all $\delta \in \mathscr{B}$. As $\mu$ is a Borel measure, $\mu$ is finite and hence $(h(N))^{1 / 2} \in L^{2}\left(\mathscr{N}, \mu_{x}\right)$. Let $U$ be the unitary transformation found in Theorem 3.4. Then if $y=U^{*} h^{1 / 2}$, using the properties of $U$,

$$
\begin{aligned}
\mu_{y}(\delta) & =\langle E(\delta) y, y\rangle \\
& =\left\langle U E(\delta) U^{*} U y, U y\right\rangle \\
& =\left\langle\chi_{\delta} h^{1 / 2}, h^{1 / 2}\right\rangle \\
& =\int_{\delta} h d \mu_{x} \\
& =\mu(\delta) .
\end{aligned}
$$

With $y$ as chosen, $y \in M(x)$ and thus $M(y) \subseteq M(x)$. Suppose the inclusion is proper and let $z \in M(x) \ominus M(y)$ and put $f=U z$. Then for all $\delta \in \mathscr{B}$,

$$
\begin{aligned}
\int_{\delta} h^{1 / 2} \bar{f} d \mu_{x} & =\left\langle U^{*} \chi_{\delta} h^{1 / 2}, U^{*} f\right\rangle \\
& =\langle E(\delta) y, z\rangle \\
& =0 .
\end{aligned}
$$

Now if $\mu \sim \mu_{x}$ then $h(N)>0 \mu_{x}$-almost everywhere and hence $\bar{f}=0$. Thus in this case, $M(x)=M(y)$.

We now prove a result which shows that $\mathscr{N}$ is a countably decomposable nest if and only if there exists a separating vector for the set of projections $\{E(\delta): \delta \in \mathscr{B}\}$.

THEOREM 3.7. If $x$ is a separating vector for $\{E(\delta): \delta \in \mathscr{B}\}$ then 
it is separating for the core $\mathscr{C}$ of the nest $\mathscr{N}$.

Proof. Suppose $x$ is not separating for $\mathscr{C}$. Then for some nonzero operator $F \in \mathscr{C}, F x=0$. As $\mathscr{C}$ is a commutative * algebra, $F$ is normal. By the spectral theorem, the spectral projections of $F$ commute with $\{F\}^{\prime}$ and hence with $\mathscr{C}^{\prime}$, and thus belong to $\mathscr{C}=\mathscr{C}^{\prime \prime}$. Hence we may assume that $F$ is a projection.

Let $y$ be a nonzero vector in the range of $F$, and let $P_{x}, P_{y}$ be the projections onto $M(x)$ and $M(y)$ respectively. Since $F E(\delta) y=$ $E(\delta) F y=E(\delta) y$, and $F E(\delta) x=E(\delta) F x=0$ for all $\delta \in \mathscr{B}$, we have that $F P_{y}=P_{y}$ and $F P_{x}=0$. By Lemma 3.3 (iv), $P_{x}, P_{y} \in \mathscr{C}^{\prime}$. Then, for all $T \in \mathscr{C}^{\prime}$, as $F \in \mathscr{C}$,

$$
\begin{aligned}
P_{y} T P_{x} & =F P_{y} T P_{x} \\
& =P_{y} T F P_{x} \\
& =0 .
\end{aligned}
$$

Similarly $P_{x} T P_{y}=0$. Also as $\mathscr{C}_{M(x)}$ has $x$ as a generating vector, by Theorem 1.1, it is maximal abelian and hence if $T, S \in \mathscr{C}^{\prime}$,

$$
P_{x} S P_{x} \cdot P_{x} T P_{x}=P_{x} T P_{x} \cdot P_{x} S P_{x} .
$$

Similar relations hold for $P_{y}$.

Now let $P=P_{x}+P_{y}$. Clearly the range of $P$ is $M(x) \oplus M(y)$. Then if $T, S \in \mathscr{C}^{\prime}$, from the previous paragraph it follows that

$$
T_{P} \cdot S_{P}=S_{P} \cdot T_{P}
$$

and hence $\left(\mathscr{C}^{\prime}\right)_{P}\left(=\left(\mathscr{C}_{P}\right)^{\prime}\right)$ is abelian. Hence $\mathscr{C}_{P}$ is a maximal abelian algebra of operators on $M(x) \oplus M(y)$. As by Corollary 3.5, $M(x) \oplus M(y)$ is separable, $\mathscr{C}_{P}$ is countably decomposable, and hence by Theorem 1.1 there exists a separating vector $z$ for $\mathscr{C}_{P}$. Considering $z$ as a vector of $H$, we have that $M(z)=M(x) \oplus M(y)$ for the projection onto the orthogonal complement of $M(z)$ in $M(x) \oplus M(y)$ is a member of $\mathscr{C}_{P}^{\prime}\left(=\mathscr{C}_{P}\right)$ (Lemma $\left.3.3(\mathrm{iv})\right)$, annihilates $z$, and so must be zero. Therefore $M(x) \subseteq M(z)$. But

$$
\begin{aligned}
\mu_{x}(\delta)=0 & \Leftrightarrow E(\delta)=0 \\
& \Leftrightarrow \mu_{z}(\delta)=0
\end{aligned}
$$

and so $\mu_{x} \sim \mu_{z}$. By considering the unitary transformation of $M(z)$ onto $L^{2}\left(\mathscr{N}, \mu_{z}\right)$, an argument identical to that in the proof of Theorem 3.6 shows that $M(z)=M(x)$. This contradicts the fact that $y \neq 0$ and thus the theorem is proved.

We shall sometimes refer to a vector that is separating for the core of a nest as a separating vector for the nest. 
If $\omega$ is any ordered set which is compact and metrisable in the order topology, by a basal measure for $\omega$ we mean a Borel measure $\mu$ on $\omega$ such that, if $a, b \in \omega$

(i) $\mu((a, b))=0$ if and only if $(a, b)$ is empty,

(ii) $\mu(a)=0$ if and only if $a=a_{-}$.

Lemma 3.8. If $\mathscr{N}$ is a countably decomposable, complete nest with core $\mathscr{C}$, then the class of measures $\left\{\mu_{x}: x\right.$ is separating for $\left.\mathscr{C}\right\}$ is an equivalence class of basal measures for $\mathscr{N}$.

Proof. If $x$ is separating for $\mathscr{C}$ and $\mu \sim \mu_{x}$ then by Theorem $3.6 \mu=\mu_{y}$ for some $y$. Let $E(\cdot)$ be the projection valued measure of $\mathscr{N}$, and let $\mathscr{B}$ be the set of Borel sets of $\mathscr{N}$. Then for all $\delta \in \mathscr{B}$,

$$
\begin{aligned}
E(\delta) y=0 & \Leftrightarrow \mu_{y}(\delta)=0 \\
& \Leftrightarrow \mu_{x}(\delta)=0 \\
& \Leftrightarrow E(\delta) x=0 \\
& \Leftrightarrow E(\delta)=0 .
\end{aligned}
$$

Therefore $y$ is separating for $\{E(\delta): \delta \in \mathscr{B}\}$, and thus by Theorem 3.7, $y$ is separating for $\mathscr{C}$. Conversely if $x$ and $y$ are separating for $\mathscr{C}$,

$$
\begin{aligned}
\mu_{x}(\delta)=0 & \Leftrightarrow E(\delta)=0 \\
\Leftrightarrow \mu_{y}(\delta) & =0
\end{aligned}
$$

and thus $\left\{\mu_{x}: x\right.$ separating for $\left.\mathscr{C}\right\}$ is an equivalence class of Borel measures. The fact that these measures are basal follow easily from the definition of $\mu_{x}$ and the fact that $x$ is separating for $\mathscr{C}$.

4. Order measure multiplicity types. For the remainder of this paper, the term nest shall be used to mean complete, countably decomposable nest, unless the contrary is explicitly stated. If $\mathscr{N}$ is a nest of subspaces of a Hilbert space $H$ with projection valued measure $E(\cdot)$, and $\delta$ is a Borel subset of $\mathscr{N}$, then we shall write $\mathscr{N}_{\delta}$ for the nest $\mathscr{N}_{E(\delta)}$.

Two vectors $x, y$ of $H$ are said to be very orthogonal (with respect to the nest $\mathscr{N}$ ) if $M(x)$ and $M(y)$ are orthogonal subspaces of $H$. This is equivalent to the statement that for all Borel subsets $\delta, \delta^{\prime}$ of $\mathscr{N},\left\langle E(\delta) x, E\left(\delta^{\prime}\right) y\right\rangle=0$, or to the statement that $x$ is orthogonal to $M(y)$. Note that this definition of very orthogonality differs from that used in [3]. If $x$ and $y$ are very orthogonal with respect to $\mathscr{N}$ then $E(\delta) x, E(\delta) y$ are very orthogonal with respect to $\mathscr{N}_{\delta}$. Conversely, if $x$ and $y$ are very orthogonal with respect to $\mathscr{N}_{\delta}$ 
then, (considered as vectors of $H$ ) they are very orthogonal with respect to $\mathscr{N}$.

If $\mathscr{N}$ is a countably decomposable nest, by Theorem 1.1 the set of vectors separating for (the core of) $\mathscr{N}$ is nonempty. A simple application of Zorn's Lemma yields a set of separating vectors for $\mathscr{N}$ which is maximal with respect to the property that any two members are very orthogonal. Such a set will be called a maximal set of very orthogonal separating vectors for $\mathscr{N}$.

Lemma 4.1. If $\Phi$ is a maximal set of very orthogonal separating vectors for a nest $\mathscr{N}$, then there exists a Borel set $\delta$ of $\mathscr{N}$ such that $E(\delta) \neq 0$ and

$$
N(\delta)=\bigoplus\{M(E(\delta) x): x \in \Phi\},
$$

where $E(\cdot)$ is the projection valued measure of $\mathscr{N}$ and $N(\delta)$ is the range of $E(\delta)$.

Proof. Let $P$ be the projection onto the subspace $\bigoplus\{M(x): x \in \Phi\}$. Then from Lemma 3.3, $P \in \mathscr{C}^{\prime}$ and thus $\mathscr{N}_{(I-P)}$ is a nest. Let $z$ be a separating vector for $\mathscr{N}_{(I-P)}$. Then $z$ (considered as a vector of $H$ ) is very orthogonal to each member of $\Phi$ and by the maximality of $\Phi$, $z$ is not separating for $\mathscr{N}$. Hence, using Theorem 3.7, there exists a Borel set $\delta$ of $\mathscr{N}$ such that

$$
\begin{aligned}
E(\delta) & \neq 0 \quad \text { and } \\
E(\delta) z & =E(\delta)(I-P) z \\
& =0 .
\end{aligned}
$$

But as $z$ is separating for $\mathscr{N}_{(I-P)}$ and $P \in \mathscr{C}^{\prime}$ this implies that $(I-P) E(\delta)=0$ and hence

$$
\begin{aligned}
N(\delta) & =N(\delta) \cap \bigoplus\{M(x): x \in \Phi\} \\
& =\bigoplus\{N(\delta) \cap M(x): x \in \Phi\} \\
& =\bigoplus\{M(E(\delta) x): x \in \Phi\},
\end{aligned}
$$

using Lemma 3.3 for the last step. This completes the proof. $|\Phi|$.

If $\Phi$ is any set, we denote the cardinality (or power) of $\Phi$ by

THEOREM 4.2. If $\Phi$ and $\Theta$ are two maximal sets of very orthogonal separating vectors for a nest $\mathscr{N}$ then

$$
|\Phi|=|\Theta| \text {. }
$$

Proof. The method of this proof is identical to that in [3], p. 
99. We divide the proof into two cases.

(i) If $|\Theta|,|\Phi|$ are both infinite.

For any vector $x$ of $H$, let $P_{x}$ be the projection onto $M(x)$. For each $x \in \Theta$ let

$$
K_{x}=\left\{y: y \in \Phi, P_{y} x \neq 0\right\} .
$$

By the maximality of $\Phi$, no member of $\Theta$ is very orthogonal to each member of $\Phi$. Thus for each $y \in \Phi$, there exists some $x \in \Theta$ such that $P_{y} x \neq 0$ or equivalently such that $y \in K_{x}$. Thus $\Phi$ is a subset of $\mathbf{U}\left\{K_{x}: x \in \Theta\right\}$. But since

$$
\|x\|^{2} \geqq \sum_{y \in \Phi}\left\|P_{y} x\right\|^{2}
$$

for each $x \in \Theta, K_{x}$ is an at most countable subset of $\Phi$. Therefore, as $|\Theta|$ is an infinite cardinal, $\left|\bigcup\left\{K_{x}: x \in \Theta\right\}\right| \leqq|\Theta|$. So we have that $|\Phi| \leqq|\Theta|$ and by symmetry, equality must hold.

(ii) If $|\Theta|,|\Phi|$ are not both infinite.

Suppose $|\Theta|=n$ is finite. We shall prove that $|\Phi| \leqq n$ and the result will then follow by symmetry. Let $\delta$ be the Borel subset of $\mathscr{N}$, provided by Lemma 4.1 such that $E(\delta) \neq 0$ and

$$
N(\delta)=\bigoplus\{M(E(\delta) x): x \in \Theta\},
$$

and let the set of vectors $\{E(\delta) x: x \in \Theta\}$ be $\left\{x_{i}: 1 \leqq i \leqq n\right\}$. Then as $\Theta$ is a set of separating vectors it follows that the measures $\left\{\mu_{x_{i}}\right\}$ are equivalent. If $\mu_{x_{1}}=\mu$, from Theorem 3.6 there exist vectors $\left\{z_{i}: 1 \leqq i \leqq n\right\}$ such that $\mu_{z_{i}}=\mu$ and $M\left(z_{i}\right)=M\left(x_{i}\right)$. Then

$$
N(\delta)=\bigoplus_{i=1}^{n} M\left(z_{i}\right)
$$

Let $U_{i}$ be the unitary transformation, provided by Theorem 3.4, of $M\left(z_{i}\right)$ onto $L^{2}(\mathscr{N}, \mu)$ and let $U=\bigoplus U_{i}$. Then $U$ takes $N(\delta)$ onto

$$
\bigoplus_{i=1}^{n} L^{2}(\mathscr{N}, \mu) \text {. }
$$

Now suppose that $|\Phi|>n$ and let $\left\{y_{i}: 1 \leqq i \leqq n+1\right\}$ be a subset of $n+1$ elements of $\{E(\delta) y: y \in \Phi\}$. For all Borel sets $\gamma$ of $\mathscr{N}$ such that $E(\delta \cap \gamma) \neq 0$, as $\Phi$ is a very orthogonal set of separating vectors, we have that $\left\langle E(\gamma) y_{i}, E(\gamma) y_{j}\right\rangle=0$ if and only if $i \neq j$. Hence

$$
\left\langle U E(\gamma) U^{*} U y_{i}, U E(\gamma) U^{*} U y_{j}\right\rangle=0
$$

if and only if $i \neq j$. Now $\varphi_{i}=U y_{i}$ is an ordered set $\left(f_{i 1}, \cdots, f_{i n}\right)$ of $n$ functions of $L^{2}(\mathscr{N}, \mu)$ and it follows from the above relations and the properties of $U$ that 


$$
\int_{\gamma} \sum_{k=1}^{n} f_{i k} \bar{f}_{j k} d \mu=0 .
$$

if and only if $i \neq j$. Hence at all points $N \in \delta$ except possibly for a $\mu$-null set, the ordered sets of complex numbers $\left(f_{i 1}(N), \cdots, f_{i n}(N)\right)$ form an orthogonal set of $n+1$ nonzero vectors of an $n$-dimensional Hilbert space. This contradiction shows that $|\Phi| \leqq n$ and the theorem follows.

For a given nest $\mathscr{N}$, we define a function $m(\cdot)$ with domain the Borel sets of $\mathscr{N}$ and range the cardinal numbers as follows. Let $E(\cdot)$ be the projection valued measure of $\mathscr{N}$ and suppose $\delta$ is a Borel subset of $\mathscr{N}$. If $E(\delta) \neq 0$, then we define $m(\delta)$ to be the cardinality of a maximal orthogonal set of separating vectors for the nest $\mathscr{N}_{\delta}$. If $E(\delta)=0, m(\delta)$ is defined to be zero. Theorem 4.2 shows that $m(\cdot)$ is uniquely defined.

Let $\omega$ be any ordered set that is compact and metrisable in the order topology and let $[\mu]$ be some equivalence class of basal measures for $\omega$. Then a function $p(\cdot)$ with domain the Borel sets of $\omega$ and range the cardinal numbers, satisfying

(i) $p(\delta)=0$ if and only if $\mu(\delta)=0$ for $\mu \in[\mu]$.

(ii) If $\Delta$ is any countable set of Borel subsets of $\omega$ such that $\mathbf{U}\{\delta: \delta \in \Delta\}$ is not $\mu$-null for $\mu \in[\mu]$, then

$$
p(\bigcup\{\delta: \delta \in \Delta\})=\min \{p(\delta): \delta \in \Delta, p(\delta) \neq 0\}
$$

is called a multiplicity function for $(\omega,[\mu])$.

Lemma 4.3. If $\mathscr{N}$ is a nest and [ $\mu$ ] is the set of measures $\left\{\mu_{x}: x\right.$ separating for $\left.\mathscr{N}\right\}$ then the function $m(\cdot)$ defined above is a multiplicity function for $(\mathscr{N},[\mu])$.

Proof. Properly (i) is obvious from the definition. If $\Delta$ is a countable set of Borel subsets of $\mathscr{N}$ let $\rho=\bigcup\{\delta: \delta \in \Delta\}$ and suppose that $\mu(\rho) \neq 0$ for some $\mu \in[\mu]$. Let $\Phi$ be a maximal set of very orthogonal separating vectors for $\mathscr{N}_{\rho}$. Then for each $\delta \in \Delta$ such that $\mu(\delta) \neq 0,\{E(\delta) x: x \in \Phi\}$ is a set of very orthogonal separating vectors for $\mathscr{N}_{\delta}$. Hence from the definition of $m(\cdot)$, if $\mu(\delta) \neq 0$,

$$
m(\rho) \leqq m(\delta) \text {. }
$$

But from Lemma 4.1, there exists a Borel set $\gamma \subseteq \rho$ such that

$$
N(\gamma)=\bigoplus\{M(E(\gamma) x): x \in \Phi\} .
$$

We then have that $m(\gamma)=m(\delta)$. Now for some member $\delta_{1}$ of $\Delta$, $\mu\left(\delta_{1} \cap \gamma\right) \neq 0$ and clearly 


$$
m\left(\delta_{1} \cap \gamma\right)=m(\gamma) .
$$

By an argument similar to that above,

$$
m\left(\delta_{1}\right) \leqq m\left(\delta_{1} \cap \gamma\right)=m(\rho)
$$

and the lemma follows.

By an order measure multiplicity set (abbreviated to o.m.m. set in the sequel), we mean a set $\{\mathscr{N},[\mu], m(\cdot)\}$ whose members satisfy the following.

I. $\mathscr{N}$ is an ordered set which is compact and metrisable in the order topology.

II. $[\mu]$ is an equivalence class of basal measures for $\mathscr{N}$.

III. $m(\cdot)$ is a multiplicity function for $(\mathscr{N},[\mu])$. If $\{\mathscr{N},[\mu], m(\cdot)\}$ is an o.m.m. set and $\delta$ is a Borel subset of $\mathscr{N}$, we call the cardinal number $m(\delta)$ the multiplicity of $\delta$. If $\delta$ has the property that for every Borel subset $\gamma$ of $\delta$ such that $\mu(\gamma) \neq 0$ we have $m(\delta)=m(\gamma)$, we say that $\delta$ has uniform multiplicity.

If $\phi$ is an order isomorphism of $\mathscr{N}$ onto another ordered set $\mathscr{C}$ then it is a homeomorphism with respect to the order topologies of $\mathscr{N}$ and $\mathscr{C}$. If $\delta$ is any subset of $\mathscr{N}$ we write $\phi(\delta)=\{\varphi(N): N \in \delta\}$. It is easy to see that if $\mathscr{B}$ denotes the Borel sets of $\mathscr{N}$, the set $\{\varphi(\delta): \delta \in \mathscr{B}\}$ is the set of Borel sets of $\mathscr{L}$. If $\{\mathscr{N},[\mu], m(\cdot)\}$ is an o.m.m. set we define

$$
\varphi(\mathscr{N},[\mu], m(\cdot)\}=\{\mathscr{K},[\nu], p(\cdot)\}
$$

where $[\nu]$ is the class of measures such that $\nu \in[\nu]$ if for some $\mu \in[\mu]$

$$
\nu(\varphi(\cdot))=\mu(\cdot),
$$

and $p(\cdot)$ is the function defined by

$$
p(\varphi(\cdot))=m(\cdot) .
$$

A simple verification shows that $\{\mathscr{C},[\nu], p(\cdot)\}$ is an o.m.m. set.

Two o.m.m. sets $\{\mathscr{N},[\mu], m(\cdot)\},\{\mathscr{K},[\nu], p(\cdot)\}$ are said to be equivalent if there exists an order isomorphism $\varphi: \mathscr{N} \rightarrow \mathscr{C l}$ such that

$$
\varphi\{\mathscr{N},[\mu], m(\cdot)\}=\{\mathscr{C},[\nu], p(\cdot)\} .
$$

It is clear that this is a genuine equivalence relation. The order isomorphism $\varphi$ is said to implement the equivalence. We call an equivalence class of o.m.m. sets an order measure multiplicity type (o.m.m. type).

The preceding results shows that every nest gives rise to a unique o.m.m. type. We shall prove that the o.m.m. type of a nest 
is a complete unitary invariant.

THEOREM 4.4. If two nests are unitarily equivalent, they determine the same o.m.m. type.

Proof. Let the two nests be $\mathscr{N}$ and $\mathscr{C l}$ and let $U$ be the unitary transformation taking $\mathscr{N}$ onto $\mathscr{C}$. Then $U$ clearly induces an order isomorphism $\varphi: \mathscr{N} \rightarrow \mathscr{l}$ where $\varphi(N)$ is the image of the subspace $N$ under $U$. If $E(\cdot), F(\cdot)$ are the projection valued measures of $\mathscr{N}$ and $\mathscr{t}$ respectively, then

$$
U E([0, N]) U^{*}=F([0, \varphi(N)])
$$

and hence for all Borel sets $\delta$ of $\mathscr{N}$,

$$
U E(\delta) U^{*}=F(\phi(\delta)) .
$$

A routine verification shows that $\Phi$ is a maximal set of very orthogonal separating vectors for $\mathscr{N}_{\delta}$ if and onlf if $\Theta=\{U x: x \in \Phi\}$ is a similar set for $\mathscr{K}_{\varphi(\delta)}$. Also if $x$ is separating for the core of $\mathscr{N}$, then $U x=y$ is separating for the core of $\mathscr{C l}$ and

$$
\begin{aligned}
\langle E(\cdot) x, x\rangle & =\left\langle U E(\cdot) U^{*} U x, U x\right\rangle \\
& =\langle F(\varphi(\cdot)) y, y\rangle .
\end{aligned}
$$

Therefore $\varphi$ implements an equivalence between the o.m.m. sets of $\mathscr{N}$ and $\mathscr{C}$ and hence $\mathscr{N}$ and $\mathscr{C}$ determine the same o.m.m. type.

5. Nests of uniform multiplicity. Let $\{\mathscr{N},[\mu], m(\cdot)\}$ be the o.m.m. set of a nest $\mathscr{N}$ of subspaces of a Hilbert space $H$. We say that the nest is of uniform multiplicity if the Borel set $\mathscr{N}$ is of uniform multiplicity for the function $m(\cdot)$. In this section we prove the converse of Theorem 4.4 for nests of uniform multiplicity. If $\mathscr{N}$ is nest of uniform multiplicity, we take $A$ as an index set with typical member $\alpha$ such that $m(\mathscr{N})=|A|$.

Before we prove the next lemma, it is of interest to show by an example that it does not hold trivially. If $r \in[0,1]$, let $V_{r}$ be the subspace of $L^{2}[0,1]$, (Lebesgue measure), consisting of functions $f$ such that $f(t)=0$ almost everywhere for $t>r$. Now let $V_{r 1}=V_{r}$ for $r<\frac{1}{2}$ and $V_{r 1}=V_{1 / 2}$ for $r \geqq \frac{1}{2}$ and for integers $i \geqq 2$ let $V_{r i}=V_{r}$. Then if

$$
N_{r}=\bigoplus_{i=1}^{\infty} V_{r i}
$$

the set $\left\{N_{r}: r \in[0,1]\right\}$ forms a complete nest $\mathscr{N}$ of subspaces of $H=$ $L^{2}\left[0, \frac{1}{2}\right] \oplus \bigoplus_{i=2}^{\infty} L^{2}[0,1]_{i}$, (where for each $i \geqq 2, L^{2}[0,1]_{i}=L^{2}[0,1]$ ). 
If $\delta$ is a Borel subset of $[0,1]$ let $\chi_{\delta} L^{2}[0,1]$ denote the subspace of $L^{2}[0,1]$ consisting of functions vanishing almost everywhere outside $\delta$. For convenience we identify a Borel set $\delta$ of $[0,1]$ with the Borel set of $\mathscr{N}$ defined by $\left\{N_{r}: r \in \delta\right\}$. Then if $E(\cdot)$ is the projection valued measure of $\mathscr{N}$, it is easily verified that $E(\delta)$ is the projection onto

$$
\chi_{\delta \cap[0,1 / 2]} L^{2}\left[0, \frac{1}{2}\right] \oplus \bigoplus_{i=2}^{\infty} \chi_{\delta} L^{2}[0,1]_{i} .
$$

Let $e_{i}$ be the unit function in $L^{2}[0,1]_{i}$ and zero elsewhere. Then the set $\left\{e_{i}: i=2,3, \cdots\right\}$ is a maximal set of very orthogonal separating vectors for $\mathscr{N}$, as no vector of $L^{2}\left[0, \frac{1}{2}\right]$ is separating for $\mathscr{N}$ and no vector of $L^{2}[0,1]_{i}$ is very orthogonal to $e_{i}$. Also, if $\delta$ is any Borel set of $[0,1]$, either the set $\left\{E(\delta) e_{i}: i=2,3, \cdots\right\}$ or this set together with $E(\delta) e_{1}$ (where $e_{1}$ is the unit function in $L^{2}\left[0, \frac{1}{2}\right]$ and zero elsewhere) forms a countable maximal set of very orthogonal separating vectors for $\mathscr{N}_{\delta}$. Hence, $\mathscr{N}$ is of uniform multiplicity. However it is easy to see that

$$
\begin{aligned}
\bigoplus_{i=2}^{\infty} M\left(e_{i}\right) & =\bigoplus_{i=2}^{\infty} L^{2}[0,1]_{i} \\
& \neq H .
\end{aligned}
$$

Therefore not every maximal set $\Phi$ of very orthogonal separating vectors for a nest $\mathscr{N}$ of uniform multiplicity generates the Hilbert space $H$ of $\mathscr{N}$ in the sense that

$$
H=\bigoplus\{M(x): x \in \Phi\} .
$$

However we have the following result.

Lemma 5.1. If $\mathscr{N}$ is a nest of subspaces of $H$ having uniform multiplicity $|A|\left(A\right.$ an index set), then there exists a set $\left\{x_{\alpha}: \alpha \in A\right\}$ of very orthogonal separating vectors such that

$$
H=\bigoplus_{\alpha \in A} M\left(x_{\alpha}\right) .
$$

Proof. Let $\mu=\mu_{x}$ for some vector $x$ that is separating for $\mathscr{N}$. Consider the set $\Delta$ of Borel sets of $\mathscr{N}$ with the properties;

(i) members of $\Delta$ are disjoint,

(ii) for each $\delta \in \Delta, \mu(\delta) \neq 0$,

(iii) for each $\delta \in \Delta$, there exists a set $\left\{x_{\alpha}^{\delta}: \alpha \in A\right\}$ of very orthogonal vectors such that

$$
N(\delta)=\bigoplus_{\alpha \epsilon_{A}} M\left(x_{\alpha}^{\delta}\right) .
$$

Lemma 4.1 shows that such sets exist and hence, by a Zorn's lemma 
argument there exists a maximal set $\Delta_{m}$ with the above properties. As $\mu$ is a finite measure, properties (i) and (ii) imply that $\Delta_{m}$ is an at most countable set, say, $\Delta_{m}=\left\{\delta_{i}: i=1,2, \cdots\right\}$. Now if $\mu\left(\mathscr{N} \backslash \bigcup_{1}^{\infty} \delta_{i}\right) \neq$ 0 then, as $\mathscr{N}$ is of uniform multiplicity, an application of Lemma 4.1 to $\mathscr{N}_{\gamma}$ where $\gamma=\mathscr{N} \backslash \bigcup_{1}^{\infty} \delta_{i}$ contradicts the maximality of $\Delta_{m}$.

It is clear that we may assume that the sets $\left\{x_{\alpha}^{i}\right\}$ satisfying (iii) for $\delta_{i}$ consist of unit vectors. Let

$$
x_{\alpha}=\sum_{i=1}^{\infty} \frac{1}{2^{i}} x_{\alpha}^{i} .
$$

It is easy to see that $\left\{x_{\alpha}\right\}$ is a set of very orthogonal separating vectors. Then, using Lemma 3.3,

$$
\begin{aligned}
\bigoplus_{\alpha \in A} M\left(x_{\alpha}\right) & =\bigoplus_{\alpha \in A} \bigoplus_{1}^{\infty} M\left(E\left(\delta_{i}\right) x_{\alpha}\right) \\
& =\bigoplus_{\alpha \in A} \bigoplus_{1}^{\infty} M\left(x_{\alpha}^{i}\right) \\
& =H .
\end{aligned}
$$

If $\delta$ is any Borel subset of $\mathscr{N}$, we denote the subspace of $L^{2}(\mathscr{N}, \mu)$ consisting of all functions vanishing $\mu$-almost everywhere on the complement of $\delta$ by the symbol $\chi_{\delta} L^{2}(\mathscr{N}, \mu)$.

THEOREM 5.2. If $\mathscr{N}$ is a nest of uniform multiplicity $|A|$ then for any vector $x$ that is separating for $\mathscr{N}$, there exists a unitary transformation from $H$ onto

$$
\bigoplus_{\alpha \in A} L^{2}\left(\mathscr{N}, \mu_{\alpha}\right)
$$

where $\mu_{\alpha}=\mu_{x}$ for all $\alpha \in A$. The image of a member $N$ of $\mathscr{N}$ under this transformation is

$$
\bigoplus_{\alpha \in A} \chi_{[0, N]} L^{2}\left(\mathscr{N}, \mu_{\alpha}\right)
$$

Proof. From Lemma 5.1,

$$
H=\bigoplus_{\alpha \in A} M\left(x_{\alpha}\right)
$$

where $\left\{x_{\alpha}: \alpha \in A\right\}$ is a set of very orthogonal separating vectors. Then for each $\alpha \in A, \mu_{x_{\alpha}} \sim \mu_{x}$ and by Theorem 3.6 there exists a vector $z_{\alpha}$ such that $M\left(z_{\alpha}\right)=M\left(x_{\alpha}\right)$ and $\mu_{z_{\alpha}}=\mu_{x}$. Hence

$$
H=\bigoplus_{\alpha \in A} M\left(z_{\alpha}\right) \text {. }
$$

Let $U_{\alpha}$ be the unitary transformation found in Theorem 3.4 taking 
$M\left(z_{\alpha}\right)$ onto $L^{2}\left(\mathscr{N}, \mu_{\alpha}\right)$ and let

$$
U=\bigoplus_{\alpha \in A} U_{\alpha}
$$

Then $U$ is a unitary transformation taking $H$ onto

$$
\bigoplus_{\alpha \in A} L^{2}\left(\mathscr{N}, \mu_{\alpha}\right)
$$

where $\mu_{\alpha}=\mu_{x}$ for all $\alpha \in A$.

If $E$ is the projection onto the member $N$ of $\mathscr{N}$, in the notation of $\S 3, E=T_{\chi_{[0, N]}}$. Hence, by Theorem 3.4, for each $a \in A$ the operator $U_{\alpha} E U_{\alpha}^{*}$ acts on $L^{2}\left(\mathscr{N}, \mu_{\alpha}\right)$ by multiplying the functions by $\chi_{[0, N]}$. Therefore, as the image of $N$ is the range of $U E U^{*}$, the theorem follows.

THEOREM 5.3. If two nests, both of uniform multiplicity, determine the same o.m.m. type then they are unitarily equivalent.

Proof. Let the nests be $\mathscr{N}$ and $\mathscr{C l}$ with o.m.m. sets $\{\mathscr{N},[\mu], m(\cdot)\},\{\mathscr{C},[\nu], p(\cdot)\}$. These o.m.m. sets belong to the same o.m.m. type and hence there exists an order isomorphism $\varphi$ implementing an equivalence between them. From the definition of equivalence $m(\mathscr{N})=p(\mathscr{C})=|A|$ where $A$ is some index set and if $\mu \in[\mu]$, there exists $\nu \in[\nu]$ such that

$$
\nu(\varphi(\cdot))=\mu(\cdot) \text {. }
$$

Since $\mathscr{N}$ and $\mathscr{C l}$ are of uniform multiplicity, Theorem 5.2 shows that they are unitarily equivalent to the nests

$$
\begin{aligned}
& \left\{\bigoplus_{\alpha \in A} \chi_{[0, N]} L^{2}\left(\mathscr{N}, \mu_{\alpha}\right): N \in \mathscr{N}\right\} \\
& \left\{\bigoplus_{\alpha \in A} \chi_{[0, M]} L^{2}\left(\mathscr{C l}, \nu_{\alpha}\right): M \in \mathscr{C}\right\}
\end{aligned}
$$

where $\mu_{\alpha}=\mu, \nu_{\alpha}=\nu$ for all $\alpha \in A$ and $\mu$ and $\nu$ satisfy the relation above. Hence it is sufficient to establish the unitary equivalence of the nests

$$
\begin{gathered}
\left\{\chi_{[0, N]} L^{2}(\mathscr{N}, \mu): N \in \mathscr{N}\right\} \\
\left\{\chi_{[0, M]} L^{2}(\mathscr{C l}, \nu): M \in \mathscr{C}\right\} .
\end{gathered}
$$

Define a transformation $U$ from $L^{2}(\mathscr{C}, \nu)$ to $L^{2}(\mathscr{N}, \mu)$ by

$$
U g(\varphi(N))=f(N) \text {. }
$$

As $\varphi$ is a homeomorphism from $\mathscr{N}$ to $\mathscr{C}$, this transformation is well defined and is clearly one to one and linear. Also if 
$g_{1}, g_{2} \in L^{2}(\mathscr{L}, \nu)$ and $U g_{1}=f_{1}, U g_{2}=f_{2}$,

$$
\begin{aligned}
\int_{\mathscr{\Lambda}} g_{1}(M) \bar{g}_{i}(\bar{M}) d \nu(M) & =\int_{\mathscr{N}} g_{1}(\varphi(N)) \bar{g}_{:}(\bar{\varphi}(\bar{N})) d \nu \varphi(N) \\
& =\int_{\mathscr{N}} f_{1}(N) \bar{f}_{2}(\bar{N}) d \mu(N)
\end{aligned}
$$

and hence $U$ is a unitary transformation. If $U g=f$ we have that $f(N)=0 \mu$-almost everywhere for $N>N_{0}$ if and only if $g(M)=0$ $\mu$-almost everywhere for $M>\varphi\left(N_{0}\right)$. Hence the two nests are unitarily equivalent under the transformation $U$.

CoROLlary 5.4. If $\mathscr{N}$ and $\mathscr{A l}$ are two nests of uniform multiplicity and $\varphi$ implements an equivalence between their o.m.m. sets then there exists a unitary transformation $U$ taking $\mathscr{N}$ onto $\mathscr{M}$ such that for each $N \in \mathscr{N}$,

$$
U N=\varphi(N) .
$$

Proof. The implementing order isomorphism was chosen arbitrarily and the unitary transformation found satisfies the required condition.

6. The general case. In order to use the results for the case of uniform multiplicity to prove the general case, it is necessary to extract from a general o.m.m. set certain Borel sets of uniform multiplicity. We do this in the two lemmas which follow.

LEMma 6.1. If $\{\mathscr{N},[\mu], m(\cdot)\}$ is any o.m.m. set and $\delta$ is a Borel set of $\mathscr{N}$ such that $m(\delta) \neq 0$, then there exists a Borel subset $\gamma$ of $\delta$ such that

(i) $\gamma$ has uniform multiplicity $m(\delta)$.

(ii) Either $m(\delta \mid \gamma)=0$ or $m(\delta \mid \gamma)>m(\delta)$.

Proof. Consider the set $\Delta$ of Borel subsets of $\delta$ defined by

$$
\Delta=\{\rho: m(\rho)>m(\delta)\} \text {. }
$$

If $\Delta$ is empty then $\delta$ has uniform multiplicity and by taking $\gamma=\delta$, the lemma follows for this case. If $\Delta$ is not empty, pick some member $\mu$ of $[\mu]$ and let

$$
a=\sup \{\mu(\rho): \rho \in \Delta\} \text {. }
$$

Now let $\left\{\rho_{i}: i=1,2, \cdots\right\}$ be a sequence of members of $\Delta$ such that

$$
\lim _{i \rightarrow \infty}\left[\mu\left(\rho_{i}\right)\right]=a .
$$


Put $\rho=\bigcup \rho_{i}$ and let $\gamma=\delta \backslash \rho$. Then as $\rho_{i} \in \Delta$ for all $i$, using the properties of the multiplicity function,

$$
\begin{aligned}
m(\delta \mid \gamma) & =m(\rho) \\
& =\min _{1 \leqq i<\infty}\left[m\left(\rho_{i}\right)\right]>m(\delta) .
\end{aligned}
$$

This shows that $\gamma$ satisfies condition (ii). Also since $m(\delta) \neq m(\rho), \gamma$ is not $\mu$-null and so

$$
m(\delta)=\min [m(\gamma), m(\rho)]=m(\gamma) .
$$

Now if $\gamma_{1}$ is any Borel subset of $\gamma$ with $\mu\left(\gamma_{1}\right) \neq 0$, then $m(\gamma)=$ $\min \left[m\left(\gamma_{1}\right), m\left(\gamma \mid \gamma_{1}\right)\right]$ and so $m\left(\gamma_{1}\right) \geqq m(\gamma)$. But if $m\left(\gamma_{1}\right)>m(\gamma)$ then $\rho \cup \gamma_{1} \in \Delta$ and $\mu\left(\rho \cup \gamma_{1}\right)>a$ contradicting the definition of $a$. Thus $m\left(\gamma_{1}\right)=m(\gamma)$ and so $\gamma$ has uniform multiplicity $m(\delta)$.

LENima 6.2. If $\{\mathscr{N},[\mu], m(\cdot)\}$ is any o.m.m. set then there exists an at most countable set $\left\{\delta_{i}: i=1,2, \cdots\right\}$ of Borel sets of $\mathscr{N}$ such that if $\mu \in[\mu]$

(i) $\mathscr{N}=\bigcup_{i=1}^{\infty} \delta_{i}$,

(ii) $\delta_{i}$ has uniform multiplicity,

(iii) $m\left(\delta_{i}\right)<m\left(\delta_{i+1}\right)$,

(iv) $\mu\left(\delta_{i} \cap \delta_{j}\right)=0$ for $i \neq j$.

Proof. For any nonzero cardinal $c$ in the range of $m(\cdot)$, by Lemma 6.1 there exists a Borel set of $\mathscr{N}$ that has uniform multiplicity $c$. It is clear that for any $\mu \in[\mu]$, any two sets of differing uniform multiplicities intersect in a $\mu$-null set. Thus the finiteness of $\mu$ implies that the range of $m(\cdot)$ is an at most countable set of cardinals $\left\{c_{i}: i=1,2, \cdots\right\}$. As the set of all cardinals is well ordered, we may index this set so that $c_{i}<c_{i+1}$.

From the properties of multiplicity functions, it is clear that $m(\mathscr{N})=c_{1}$. We define inductively a sequence of Borel sets of $\mathscr{N}$. Let $\delta_{1}$ be a set of uniform multiplicity $c_{1}$ obtained from $\mathscr{N}$ as in Lemma 6.1, and for each integer $k$, let $\delta_{k}$ be a set obtained in the same way from $\mathscr{N} \backslash \bigcup_{i=1}^{k-1} \delta_{i}$ providing this set is not of uniform multiplicity, and let $\delta_{k}=\mathscr{N} \backslash \bigcup_{i=1}^{k-1} \delta_{i}$ if this set is of uniform multiplicity. In the latter case the process terminates with $\delta_{k}$. That the set $\left\{\delta_{i}: i=1,2, \cdots\right\}$ has properties (ii) and (iii) follow directly from properties (i) and (ii) of Lemma 6.1 and the indexing of the range of $m(\cdot)$. Property (iv) is obvious from the construction, as the sets are disjoint.

If the process terminates, property (i) follows from the construction. If the process does not terminate, we prove that 


$$
\mu\left(\mathscr{N} \mid \bigcup_{1}^{\infty} \delta_{i}\right)=0
$$

For if this is false, then for some integer $k$,

$$
m\left(\mathscr{N} \backslash \bigcup_{1}^{\infty} \delta_{i}\right)=c_{k} \text {. }
$$

From the construction and property (ii) of Lemma 6.1, $m\left(\mathscr{N} \backslash \bigcup_{1}^{k} \delta_{i}\right)=$ $c_{k+1}$. But

$$
\mathscr{N} \mid \bigcup_{1}^{k} \delta_{i}=\left(\mathscr{N} \mid \bigcup_{1}^{\infty} \delta_{i}\right) \cup \bigcup_{k+1}^{\infty} \delta_{i}
$$

and so as $m(\cdot)$ is a multiplicity function we also have that

$$
m\left(\mathscr{N} \backslash \bigcup_{1}^{k} \delta_{i}\right) \leqq c_{k}
$$

Therefore $\mu\left(\mathscr{N} \backslash \bigcup_{1}^{\infty} \delta_{i}\right)=0$ and putting $\delta_{1} \cup\left(\mathscr{N} \backslash \bigcup_{1}^{\infty} \delta_{i}\right)$ in place of $\delta_{1}$ in the set $\left\{\delta_{i}: i=1,2, \cdots\right\}$ does not destroy properties (i), (iii) or (iv). The set then also satisfies (i).

Lemma 6.3. Suppose two nests $\mathscr{N}$ and $\mathscr{A l}$ determine equivalent o.m.m. sets $\{\mathscr{N},[\mu], m(\cdot)\}, \quad\{\mathscr{C}[\nu], p(\cdot)\}$ and $\varphi$ implements this equivalence. Let $\delta$ be a Borel set of $\mathscr{N}$ having uniform multiplicity. Then if $\varphi(\delta)=\gamma$, the nests $\mathscr{N}_{\delta}, \mathscr{L}_{\gamma}$ determine equivalent o.m.m. sets.

Proof. The difficulties of this proof are largely notational. Let $E(\cdot)$ be the projection valued measure of $\mathscr{N}$ and for each $N \in \mathscr{N}$, denote by $N_{\delta}$ the range of $E([0, N] \cap \delta)$ regarded as a subspace of the range of $E(\delta)$. For each $M \in \mathscr{C}$, we define $M_{\gamma}$ in a similar way. Then the nests $\mathscr{N}_{\delta}, \mathscr{L}_{\gamma}$ are $\left\{N_{\delta}: N \in \mathscr{N}\right\}$ and $\left\{M_{\gamma}: M \in \mathscr{L}\right\}$ respectively. Let $\psi$ be a map from $\mathscr{N}_{\delta}$ onto $\mathscr{L}_{\gamma}$ defined by

$$
\psi\left(N_{\delta}\right)=[\varphi(N)]_{\gamma} .
$$

This map is clearly order preserving. Also, if $N_{\delta}, N_{\delta}^{\prime}$ are distinct members of $\mathscr{N}_{\delta}$ then $E\left(\left(N, N^{\prime}\right] \cap \delta\right) \neq 0$ and hence for $\mu \in[\mu]$, $\mu\left(\left(N, N^{\prime}\right] \cap \delta\right) \neq 0$. Therefore, as for each $\nu \in[\nu], \nu(\varphi(\cdot))=\mu(\cdot)$ for some $\mu \in[\mu]$, if $\varphi(N)=M, \varphi\left(N^{\prime}\right)=M^{\prime}, \nu\left(\left(M, M^{\prime}\right] \cap \gamma\right) \neq 0$. Hence if $F(\cdot)$ is the projection valued measure of $\mathscr{C}, F\left(\left(M, M^{\prime}\right] \cap \gamma\right) \neq 0$ and thus $M_{\gamma}, M_{\gamma}^{\prime}$ are distinct. Therefore $\psi$ is an order isomorphism between $\mathscr{N}_{\delta}$ and $\mathscr{M}_{\gamma}$.

Let $\mathscr{B}$ be the set of Borel sets of $\mathscr{N}$. If $\beta \in \mathscr{B}$ let $\beta^{\prime}$ be the subset of $\mathscr{N}_{\delta}$ defined by $\left\{N_{\delta}: N \in \beta\right\}$. The set $\left\{\beta^{\prime}: \beta \in \mathscr{B}\right\}$ is clearly a $\sigma$-ring containing the open order intervals of $\mathscr{N}_{\delta}$ and hence contains 
the Borel sets of $\mathscr{N}_{\delta}$. Now if $E^{\prime}(\cdot)$ is the projection valued measure of $\mathscr{N}_{\delta}$, a routine verification shows that

$$
E^{\prime}\left(\beta^{\prime}\right)=E(\beta)_{E(\delta)} .
$$

If $x$ is separating for $\mathscr{N}$ then $E(\delta) x$ is separating for $\mathscr{N}_{\delta}$ and thus, by Lemma 3.8, the set of measures $\left[\mu^{\prime}\right]$ of the o.m.m. set of $\mathscr{N}_{\delta}$ is the equivalence class of $\mu_{x}^{\prime}$ where

$$
\begin{aligned}
\mu_{x}^{\prime}\left(\beta^{\prime}\right) & =\left\langle E^{\prime}\left(\beta^{\prime}\right) E(\delta) x, E(\delta) x\right\rangle \\
& =\mu_{x}(\delta \cap \beta) .
\end{aligned}
$$

Therefore, for each $\mu^{\prime} \in\left[\mu^{\prime}\right]$ there exists $\mu \in[\mu]$ such that $\mu^{\prime}\left(\beta^{\prime}\right)=\mu(\delta \cap \beta)$. A similar result holds for $\mathscr{C}_{\gamma}$. Hence, if $\left[\nu^{\prime}\right]$ is the set of measures of the o.m.m. set of $\mathscr{L}_{\gamma}$,

$$
\begin{aligned}
\mu^{\prime}\left(\beta^{\prime}\right) & =\mu(\beta \cap \delta) \\
& =\nu(\varphi(\beta) \cap \gamma) \\
& =\nu^{\prime}\left(\varphi(\beta)^{\prime}\right)
\end{aligned}
$$

where $\varphi(\beta)^{\prime}=\left\{M_{\gamma}: M \in \varphi(\beta)\right\}$. But then

$$
\begin{aligned}
\varphi(\beta)^{\prime} & =\left\{\psi\left(N_{\delta}\right): N \in \beta\right\} \\
& =\psi\left(\beta^{\prime}\right)
\end{aligned}
$$

and thus for each $\mu^{\prime} \in\left[\mu^{\prime}\right]$ there exists $\nu^{\prime} \in\left[\nu^{\prime}\right]$ with $\mu^{\prime}(\cdot)=\nu^{\prime}(\psi(\cdot))$.

As $\delta$ is a set of uniform multiplicity it is easy to see that $\mathscr{N}_{\delta}$ is a nest of uniform multiplicity and as $m(\cdot)=p(\varphi(\cdot))$, $\mathscr{C}_{\gamma}$ is a nest of the same uniform multiplicity. Hence the o.m.m. sets of $\mathscr{N}_{\delta}$ and $\mathscr{C l}_{\gamma}$ are equivalent.

Theorem 6.4. If two nests $\mathscr{N}$ and $/ l$ determine the same o.m.m. type then they are unitarily equivalent.

Proof. Let $\varphi$ be the order isomorphism implementing the equivalence of the o.m.m. sets of $\mathscr{N}$ and $\mathscr{C l}$ and let $\left\{\delta_{i}: i=1,2, \cdots\right\}$ be Borel sets of $\mathscr{N}$ of uniform multiplicity, obtained from the o.m.m. set of $\mathscr{N}$ as in Lemma 6.2. Then if $\gamma_{i}=\varphi\left(\delta_{i}\right),\left\{\gamma_{i}: i=1,2, \cdots\right\}$ are Borel sets of th having similar properties. By Lemma 6.3, for each $i$ the o.m.m. sets of $\mathscr{N}_{\delta_{i}}, \mathscr{C}_{\gamma_{i}}$ are equivalent and as these nests are of uniform multiplicity, by Theorem 5.3 there exists a unitary transformation $U_{i}$ taking $\mathscr{N}_{\delta_{i}}$ onto $\mathscr{M}_{\gamma_{i}}$.

Let $E(\cdot)$ be the projection valued measure of $\mathscr{N}$ and for each $N \in \mathscr{N}$ let $N_{i}$ be the range of $E\left([0, N] \cap \delta_{i}\right)$. Define $M_{i}$ similarly for each $M \in \mathscr{l l}$. Then from the proof of Lemma 6.3, the map $\psi_{i}$ from $\mathscr{N}_{\delta_{i}}$ onto $\mathscr{C}_{\gamma_{i}}$ defined by $\psi_{i}\left(N_{i}\right)=\varphi(N)_{i}$ implements the 
equivalence between the o.m.m. sets of $\mathscr{N}_{\delta_{i}}$ and $\mathscr{L}_{\gamma_{i}}$. Hence by Corollary 5.4 we may suppose that $U_{i} N_{i}=M_{i}$ where $M=\varphi(N)$.

As $\mathscr{N}=\bigcup_{1}^{\infty} \delta_{i}$, for each $N \in \mathscr{N}$,

$$
N=\bigoplus_{1}^{\infty} N_{i}
$$

If $U=\bigoplus_{1}^{\infty} U_{i}$, we have that if $\varphi(N)=M$,

$$
\begin{aligned}
U N & =\bigoplus_{1}^{\infty} U_{i} N_{i} \\
& =\bigoplus_{1}^{\infty} M_{i} \\
& =M
\end{aligned}
$$

and hence the nests $\mathscr{N}$ and $\mathscr{C}$ are unitarily equivalent.

In the theorem below we complete the proof that the class of o.m.m. types form a complete set of invariants by constructing, for an arbitrary o.m.m. type a (complete and countably decomposable) nest which gives rise to it. We then also have a typical member of each unitary equivalence class of nests. This typical nest, as it depends on the choice of an o.m.m. set from the given o.m.m. type, is not unique. It follows from Theorem 2.2 that it is possible to choose, from any o.m.m. type, an o.m.m. set whose ordered set is a closed subset of $[0,1]$. The typical nest corresponding to this o.m.m. set may be useful in applications.

THeOREM 6.5. For any o.m.m. type, there exists a nest which gives rise to it.

Proof. Let $\{\mathscr{N},[\mu], m(\cdot)\}$ be some member of the given o.m.m. type and let $\left\{\delta_{i}: i=1,2, \cdots\right\}$ be a set of Borel sets obtained from $\{\mathscr{N},[\mu], m(\cdot)\}$ as in Lemma 6.2. Define a set $\left\{\beta_{i}: i=1,2, \cdots\right\}$ of Borel sets of $\mathscr{N}$ by $\beta_{1}=\mathscr{N}$ and $\beta_{i+1}=\beta_{i} \mid \delta_{i}$. Let $\mu_{1} \in[\mu]$ and define $\mu_{i}$ by

$$
\mu_{i}(\delta)=\mu_{1}\left(\delta \cap \beta_{i}\right)
$$

for all Borel sets $\delta$ of $\mathscr{N}$. From Lemma 6.2 (iii), $\left\{m\left(\delta_{i}\right): i=1,2, \cdots\right\}$ is a set of cardinals increasing with $i$ and we may thus pick suitable index sets $A_{i}$ with typical members $\alpha_{i}$ such that $\left|A_{1}\right|=m\left(\delta_{1}\right)$ and $\left|A_{i}\right|=m\left(\delta_{i}\right)-m\left(\delta_{i-1}\right)$. For each $N \in \mathscr{N}$ define $N^{\prime}$ by

$$
N^{\prime}=\bigoplus_{i=1}^{\infty} \bigoplus_{\alpha_{i} \in A_{i}} \chi_{[0, N]} L^{2}\left(\mathscr{N}, \mu_{\alpha_{i}}\right)
$$

where $\mu_{\alpha_{i}}=\mu_{i}$ for all $\alpha_{i} \in A_{i}$. Consider the set $\left\{N^{\prime}: N \in \mathscr{N}\right\}$. We 
shall prove that this is a complete and countably decomposable nest and gives rise to the given o.m.m. type.

It is clear that $\mathscr{N}^{\prime}$ is a totally ordered set of subspaces of a Hilbert space. We first show that it is order isomorphic to $\mathscr{N}$. Let $\varphi$ be the map from $\mathscr{N}$ onto $\mathscr{N}^{\prime}$ defined by $\varphi(N)=N^{\prime}$. Then $\varphi$ is order preserving. Also if $N_{1}$ and $N_{2}$ are distinct members of $\mathscr{N}$, say $N_{1}<N_{2}$, then as $\mu_{1}$ is a basal measure for $\mathscr{N}, \mu_{1}\left(\left(N_{1}, N_{2}\right]\right) \neq 0$ for either $N_{1}=N_{2-}$ or the interval $\left(N_{1}, N_{2}\right)$ is nonvoid. Hence

$$
N_{2}^{\prime} \ominus N_{1}^{\prime}=\bigoplus_{1}^{\infty} \bigoplus_{\alpha_{i} \in A_{i}} \chi_{\left(N_{1}, N_{2}\right]} L^{2}\left(\mathscr{N}, \mu_{\alpha_{i}}\right)
$$

is nonzero and $\varphi$ is thus an order isomorphism.

We now show that the nest $\mathscr{N}^{\prime}$ is complete. Let $\Lambda^{\prime}$ be any subset of $\mathscr{N}^{\prime}$ and let $\Lambda$ be the corresponding subset of $\mathscr{N}$. Suppose that

$$
N_{0}=\sup \{N: N \in \Lambda\} .
$$

If the supremum is attained, it is clear that

$$
N_{0}^{\prime}=\bigcup\left\{N^{\prime}: N^{\prime} \in \Lambda^{\prime}\right\} \text {. }
$$

If the supremum is not attained, then $N_{0}=N_{0^{-}}$and as $\mu_{1}$ is a basal measure, $\mu_{1}\left(\left\{N_{0}\right\}\right)=0$. Since $\mathscr{N}$ is metrisable in the order topology, there exists an increasing sequence $\left\{N_{k}\right\}$ of points of $\mathscr{N}$ such that $\sup _{k}\left(N_{k}\right)=N_{0}$. For any $f \in \chi_{\left[0, N_{0}\right]} L^{2}\left(\mathscr{N}, \mu_{i}\right)$ let $f_{k}=\chi_{\left[0, N_{k}\right]} f$. Then

$$
\begin{aligned}
\left\|f-f_{k}\right\|^{2} & =\int_{\mathscr{S}} \chi_{\left(N_{k}, N_{0}\right]}|f|^{2} d \mu_{i} \\
& =\int_{\mathscr{S}} \chi_{\left(N_{k}, N_{0}\right)}|f|^{2} d \mu_{i}
\end{aligned}
$$

since $\mu\left(\left\{N_{0}\right\}\right)=0$. But $\chi_{\left(N_{k}, N_{0}\right)}$ tends to zero pointwise, and $\chi_{\left(N_{k}, N_{0}\right)}|f|^{2} \leqq|f|^{2}$, hence by the dominated convergence theorem (see e.g. [2] p. 110)

$$
\left\|f-f_{k}\right\|^{2} \rightarrow 0 \text {. }
$$

Therefore it is easy to see that

$$
\operatorname{cl}\left[\bigcup\left\{N^{\prime}: N^{\prime} \in \Lambda^{\prime}\right\}\right]=N_{0}^{\prime} .
$$

A routine verification shows that the intersection of any set of members of $\mathscr{N}^{\prime}$ is also a member of $\mathscr{N}^{\prime}$.

As $\mathscr{N}^{\prime}$ is complete and is metrisable in the order topology, the projection valued measure $E^{\prime}(\cdot)$ of $\mathscr{N}^{\prime}$ can be set up as in $\S 3$. It is easy to see that if $\delta$ is a Borel set of $\mathscr{N}$, the range of $E^{\prime}(\varphi(\delta))$ is 


\section{$\bigoplus_{i=1}^{\infty} \bigoplus_{\alpha_{i} \in A_{i}} \chi_{\delta} L^{2}\left(\mathscr{N}, \mu_{\alpha_{i}}\right)$}

Let $x_{1}$ be the vector that is the unit function in $L^{2}\left(\mathscr{N}, \mu_{\alpha_{i}}\right)$ for some $\alpha_{1} \in A_{1}$ and zero elsewhere. Since $E^{\prime}(\varphi(\delta)) x_{1}$ is the function $\chi_{\delta}$ in $L^{2}\left(\mathscr{N}, \mu_{\alpha_{1}}\right)$ and zero elsewhere, it is easy to see that $x_{1}$ is separating for the set of projections $\left\{E^{\prime}(\varphi(\delta)): \delta\right.$ a Borel set of $\left.\mathscr{N}\right\}$. Hence, as $\varphi$ is a homeomorphism, using Theorems 1.1 and 3.7 it follows that $\mathscr{N}^{\prime}$ is countably decomposable. Also for each Borel set $\delta$ of $\mathscr{N}$,

$$
\left\langle E^{\prime}(\varphi(\delta)) x_{1}, x_{1}\right\rangle=\mu_{1}(\delta)
$$

and therefore the class of measures of the o.m.m. set of $\mathscr{N}^{\prime \prime}$ is an equivalence class $[\nu]$ such that for each $\nu \in[\nu]$, there exists $\mu \in[\mu]$ with

$$
\nu(\varphi(\cdot))=\mu(\cdot) .
$$

Let $p(\cdot)$ be the multiplicity function of $\mathscr{N}^{\prime}$. It remains to prove that $p(\varphi(\cdot))=m(\cdot)$. For each integer $k$ let $\gamma_{k}=\varphi\left(\delta_{k}\right)$, where $\left\{\delta_{i}: i=1,2, \cdots\right\}$ are the sets introduced previously. We show first that $m\left(\delta_{k}\right)=p\left(\gamma_{k}\right)$. Let $x_{\alpha_{i}}$ be the vector that is the unit function in $L^{2}\left(\mathscr{N}, \mu_{\alpha_{i}}\right)$ and zero elsewhere. Put $z_{\alpha_{i}}=E^{\prime}\left(\gamma_{k}\right) x_{\alpha_{i}}$. Then $\left\|z_{\alpha_{i}}\right\|^{2}=$ $\mu_{\alpha_{i}}\left(\delta_{k}\right)=\mu_{1}\left(\delta_{i} \cap \delta_{k}\right)$, and thus from the definition of $\gamma_{i}, z_{\alpha_{i}}=0$ for $i>k$. Clearly the set $\Phi=\left\{z_{\alpha_{i}}: \alpha_{i} \in A_{i}, i=1,2, \cdots\right\}$ is a very orthogonal set for $\mathscr{N}_{\gamma_{k}}^{\prime}$. It is easy to verify that each member of is olso separating for $\mathscr{N}_{\gamma_{k}}^{\prime}$. As the range of $E^{\prime}\left(\gamma_{k}\right)$ is

$$
\bigoplus_{i=1}^{k} \bigoplus_{\alpha_{i} \in A_{i}} \chi_{\delta_{k}} L^{2}\left(\mathscr{N}, \mu_{\alpha_{i}}\right)
$$

and $M\left(z_{\alpha_{i}}\right)=\chi_{\delta_{k}} L^{2}\left(\mathscr{N}, \mu_{\alpha_{i}}\right), \Phi$ is a maximal set of very orthogonal separating vectors. This also shows that $\gamma_{k}$ is a set of uniform multiplicity. Now

$$
\begin{aligned}
|\Phi| & =\sum_{i=1}^{k}\left|A_{i}\right| \\
& =m\left(\delta_{1}\right)+\sum_{i=2}^{k}\left|m\left(\delta_{i}\right)-m\left(\delta_{i-1}\right)\right| \\
& =m\left(\delta_{k}\right) .
\end{aligned}
$$

Thus $\quad m\left(\delta_{k}\right)=p\left(\gamma_{k}\right)$.

Now if $\delta$ is any Borel set of $\mathscr{N}$, as the range of $m(\cdot)$ is $\left\{m\left(\delta_{i}\right): i=1,2, \cdots\right\}$, for some $k, m(\delta)=m\left(\delta_{k}\right)$. By property (ii) of multiplicity functions, if $\mu_{1}\left(\delta \cap \delta_{i}\right) \neq 0$,

$$
m(\delta) \leqq m\left(\delta \cap \delta_{i}\right)=m\left(\delta_{i}\right)
$$


and hence for $i<k, \quad \mu_{1}\left(\delta \cap \delta_{i}\right)=0$. Also if $\mu_{1}\left(\delta \cap \delta_{k}\right)=0$, by properties (i) and (iii) of Lemma 6.2,

$$
\begin{aligned}
m(\delta) & =\min \left\{m\left(\delta \cap \delta_{i}\right): i>k\right\} \\
& >m\left(\delta_{k}\right)
\end{aligned}
$$

Hence $\mu_{1}\left(\delta \cap \delta_{k}\right) \neq 0$. Now if $\gamma=\varphi(\delta)$ and if $\nu_{1}(\cdot)=\mu_{1}(\varphi(\cdot)), \nu_{1}\left(\gamma \cap \gamma_{i}\right)=$ 0 for $i<k$ and $\nu_{1}\left(\gamma \cap \gamma_{k}\right) \neq 0$. Hence, since $\nu_{1}$ is a measure of the o.m.m. set of $\mathscr{N}^{\prime}$ and $\mathscr{N}^{\prime}=\bigcup_{1}^{\infty} \gamma_{i}$,

$$
\begin{aligned}
p(\gamma) & =\min \left\{p\left(\gamma \cap \gamma_{i}\right): \nu_{1}\left(\gamma \cap \gamma_{i}\right) \neq 0\right\} \\
& =p\left(\gamma \cap \gamma_{k}\right) \\
& =p\left(\gamma_{k}\right) \\
& =m\left(\delta_{k}\right) \\
& =m(\delta),
\end{aligned}
$$

using the fact that $\gamma_{k}$ is of uniform multiplicity. This completes the proof.

I wish to thank Professor J. R. Ringrose for his encouragement and for many helpful suggestions during the preparation of this paper. Added February 2 1967: The question referred to at the end of Theorem 2.2 has been settled by J. R. Ringrose who has constructed a nest which is metrisable in the order topology but is not countably decomposable.

\section{REFERENCES}

1. J. Dixmier, Les algèbres d'operateurs dans l'espace Hilbertien, Gauthier-Villars, Paris, 1957.

2. P. R. Halmos, Measure Theory, Van Nostrand, Princeton, 1950.

3. - Introduction to Hilbert Space, Chelsea, New York, 1951.

4. R. V. Kadison and I. M. Singer, Triangular Operator Algebras, Amer. J. Math. 82 (1960), 227-259.

5. E. Kamke, Theory of Sets, Dover, New York, 1950.

6. J. L. Kelley, General Topology, Van Nostrand, Princeton, 1955.

7. J. R. Ringrose, Superdiagonal forms for compact linear operators, Proc. London Math. Soc. (3) 12 (1962), 367-384.

8. - On some algebras of operators, Proc. London Math. Soc. (3) 15 (1965), $61-83$.

Received May 8, 1965.

GLASGOW, UNIVERSITY

GLASGOW, W. 2.

Present Address: King'S CoLlege, LONDON. 


\section{PACIFIC JOURNAL OF MATHEMATICS}

\section{EDITORS}

\section{H. ROYDEN \\ Stanford University \\ Stanford, California}

\author{
J. P. JANS \\ University of Washington \\ Seattle, Washington 98105
}

J. DugundJI

Department of Mathematics

Rice University

Houston, Texas 77001

RichaRd ARENS

University of California

Los Angeles, California 90024

\section{ASSOCIATE EDITORS}
E. F. BECKENBACH
B. H. NeumanN
F. WOLF
K. YOSIDA

\section{SUPPORTING INSTITUTIONS}

\author{
UNIVERSITY OF BRITISH COLUMBIA \\ CALIFORNIA INSTITUTE OF TECHNOLOGY \\ UNIVERSITY OF CALIFORNIA \\ MONTANA STATE UNIVERSITY \\ UNIVERSITY OF NEVADA \\ NEW MEXICO STATE UNIVERSITY \\ OREGON STATE UNIVERSITY \\ UNIVERSITY OF OREGON \\ OSAKA UNIVERSITY \\ UNIVERSITY OF SOUTHERN CALIFORNIA
}

\author{
STANFORD UNIVERSITY \\ UNIVERSITY OF TOKYO \\ UNIVERSITY OF UTAH \\ WASHINGTON STATE UNIVERSITY \\ UNIVERSITY OF WASHINGTON

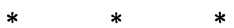 \\ AMERICAN MATHEMATICAL SOCIETY \\ CHEVRON RESEARCH CORPORATION \\ TRW SYSTEMS \\ NAVAL ORDNANCE TEST STATION
}

Mathematical papers intended for publication in the Pacific Journal of Mathematics should be typewritten (double spaced). The first paragraph or two must be capable of being used separately as a synopsis of the entire paper. It should not contain references to the bibliography. Manuscripts may be sent to any one of the four editors. All other communications to the editors should be addressed to the managing editor, Richard Arens at the University of California, Los Angeles, California 90024.

50 reprints per author of each article are furnished free of charge; additional copies may be obtained at cost in multiples of 50 .

The Pacific Journal of Mathematics is published monthly. Effective with Volume 16 the price per volume (3 numbers) is $\$ 8.00$; single issues, $\$ 3.00$. Special price for current issues to individual faculty members of supporting institutions and to individual members of the American Mathematical Society: $\$ 4.00$ per volume; single issues $\$ 1.50$. Back numbers are available.

Subscriptions, orders for back numbers, and changes of address should be sent to Pacific Journal of Mathematics, 103 Highland Boulevard, Berkeley 8, California.

Printed at Kokusai Bunken Insatsusha (International Academic Printing Co., Ltd.), 7-17, Fujimi 2-chome, Chiyoda-ku, Tokyo, Japan.

PUBLISHED BY PACIFIC JOURNAL OF MATHEMATICS, A NON-PROFIT CORPORATION

The Supporting Institutions listed above contribute to the cost of publication of this Journal, but they are not owners or publishers and have no responsibility for its content or policies. 


\section{Pacific Journal of Mathematics}

\section{Vol. 23, No. 2 \\ April, 1967}

Herbert Stanley Bear, Jr. and Bertram John Walsh, Integral kernel for

one-part function spaces .......................... 209

Mario Borelli, Some results on ampleness and divisorial schemes ....... 217

John A. Erdos, Unitary invariants for nests . ................... 229

Nathaniel Grossman, The volume of a totally-geodesic hypersurface in a pinched manifold.................................. 257

D. M. Hyman, A generalization of the Borsuk-Whitehead-Hanner theorem ............................................. 263

I. Martin (Irving) Isaacs, Finite groups with small character degrees and large prime divisors ............................. 273

I. Martin (Irving) Isaacs, Two solvability theorems ................ 281

William Lee Johnson, The characteristic function of a harmonic function in a locally Euclidean space ............................... 291

Ralph David Kopperman, Application of infinitary languages to metric spaces ............................................. 299

John Lauchlin MacDonald, Relative functor representability ............ 311

Mahendra Ganpatrao Nadkarni, A class of measures on the Bohr group.... 321

Keith Lowell Phillips, Hilbert transforms for the p-adic and p-series fields....................................... 329

Norman R. Reilly and Herman Edward Scheiblich, Congruences on regular semigroups ...

Neil William Rickert, Measures whose range is a ball ... . .

Gideon Schwarz, Variations on vector measures

Ronald Cameron Riddell, Spectral concentration for self-adjoint operators. .

Haskell Paul Rosenthal, A characterization of restrictions of

Fourier-Stieltjes transforms ................... 